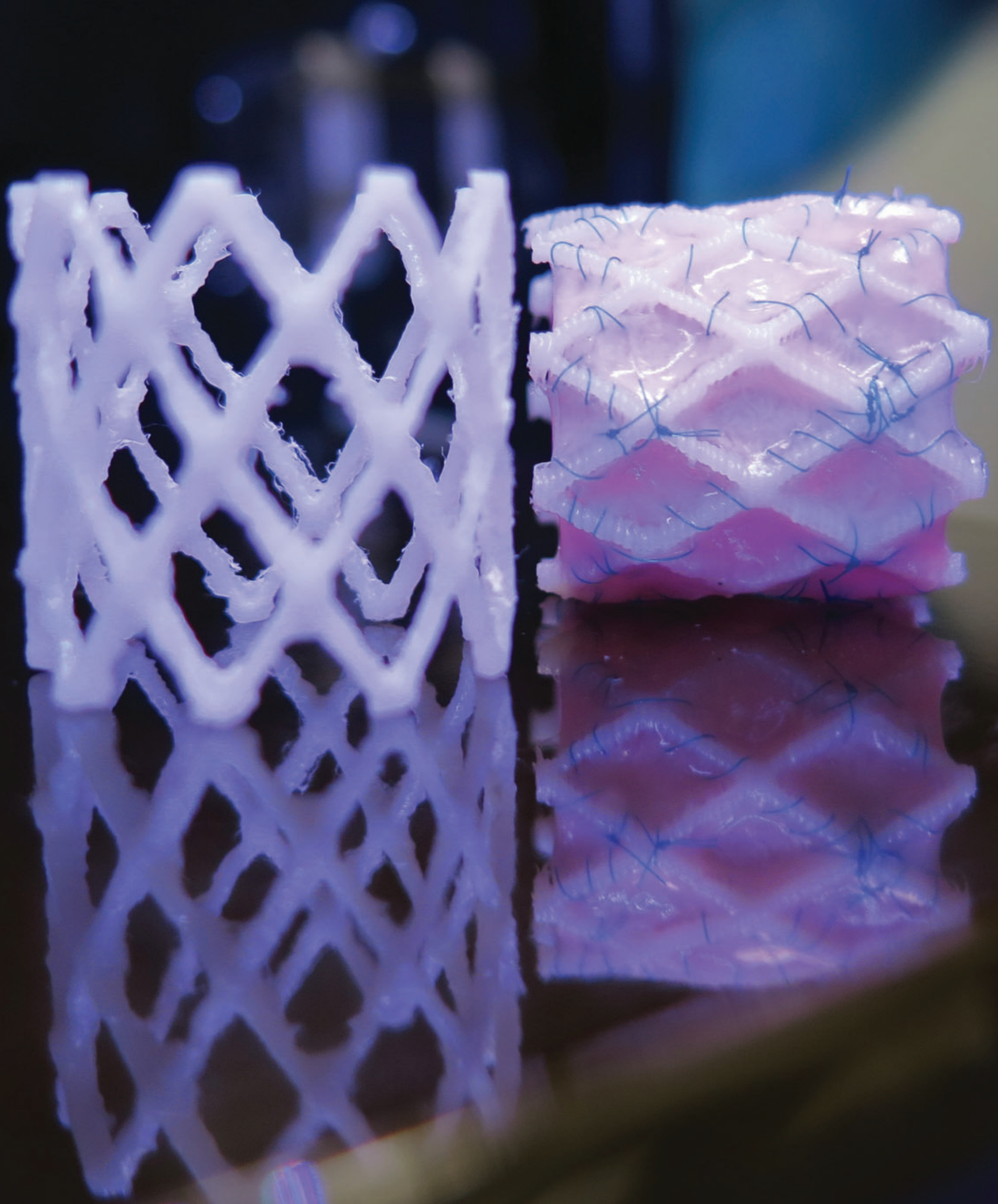




\title{
Computationally Designed 3D Printed Self-Expandable Polymer Stents with Biodegradation Capacity for Minimally Invasive Heart Valve Implantation: A Proof-of-Concept Study
}

\author{
María Sol Cabrera, ${ }^{1, *}$ Bart Sanders, ${ }^{1,2, *}$ Olga J.G.M. Goor, ${ }^{2,3}$ Anita Driessen-Mol,
} Cees W.J Oomens, ${ }^{1,2}$ and Frank P.T. Baaijens ${ }^{1,2}$

\begin{abstract}
The evolution of minimally invasive implantation procedures and the in vivo remodeling potential of decellularized tissue-engineered heart valves require stents with growth capacity to make these techniques available for pediatric patients. By means of computational tools and 3D printing technology, this proof-ofconcept study demonstrates the design and manufacture of a polymer stent with a mechanical performance comparable to that of conventional nitinol stents used for heart valve implantation in animal trials. A commercially available 3D printing polymer was selected, and crush and crimping tests were conducted to validate the results predicted by the computational model. Finally, the degradability of the polymer was assessed via accelerated hydrolysis.
\end{abstract}

Keywords: biodegradable polymer stent, computational modeling, minimally invasive surgery, tissueengineered heart valve replacement

\section{Introduction}

EVERY YEAR, $\sim 280,000$ patients undergo heart valve surgery worldwide ${ }^{1}$ and the number of patients requiring heart valve replacement is expected to triple by $2050 .^{2}$ Bioprosthetic and mechanical valves are currently available lifesaving substitutes. ${ }^{3}$ Nonetheless, the lack of repair and growth capabilities of these alternatives demands staged interventions in pediatric patients as they outgrow the prostheses. Recent developments in decellularized tissueengineered valves (DTEHVs) are promising in terms of remodeling capacity. These valves were implanted mini- mally invasively in sheep and demonstrated rapid host cell repopulation and extracellular matrix production, indicative for growth potential. ${ }^{4}$ Stent devices for minimally invasive heart valve implantation are crucial to enable delivery and provide instant support to the prosthesis after deployment. However, the stent is only required until the heart valve is fully integrated into the host tissue. In addition, for DTEHVs to be considered as an alternative for pediatric patients, it is of importance that the stent can accommodate for growth. The currently used metal stents, which reside permanently inside the body, lack growth capacity and may incur longterm complications such as hyperplasia. ${ }^{5,6}$ Bioabsorbable

\footnotetext{
${ }^{1}$ Department of Biomedical Engineering, Group of Soft Tissue Biomechanics and Tissue Engineering, Eindhoven University of Technology, Eindhoven, the Netherlands.

${ }^{2}$ Institute for Complex Molecular Systems, Eindhoven University of Technology, Eindhoven, the Netherlands.

${ }^{3}$ Department of Biomedical Engineering, Group of Biomedical Chemistry, Eindhoven University of Technology, Eindhoven, the Netherlands.

*These authors contributed equally to this work.

Opposite page: 3D printed biodegradable stent for tissue-engineered heart valves. Photo credit: Dr. B. Sanders and Dr. M.S. Cabrera, Eindhoven University of Technology.

(C) María Sol Cabrera, et al., 2017; Published by Mary Ann Liebert, Inc. This Open Access article is distributed under the terms of the Creative Commons Attribution Noncommercial License (http://creativecommons.org/licenses/by-nc/4.0/) which permits any noncommercial use, distribution, and reproduction in any medium, provided the original author(s) and the source are credited.
} 
polymers, broadly investigated for their applicability in stent devices, are an attractive option. ${ }^{7}$ These materials are not only of interest due to their short-term presence but also because they might enable other benefits, such as low late thrombus formation and less interference with minimally invasive techniques such as magnetic resonance imaging. ${ }^{8}$

Polymer stents for minimally invasive heart valve applications have to meet a set of criteria such as sufficient radial force (RF), to maintain their position after deployment, and limited plastic deformation to secure initial valve functioning until host body integration. In this proof-of-concept study, we aim to produce a computational-based, self-expandable polymer stent prototype with biodegradation potential. Our goal is to achieve the same mechanical performance and implantation capacity as a nitinol stent currently used for preclinical implantation studies of DTEHVs in sheep. ${ }^{4}$ To design a polymer stent with such requirements, a finite element (FE) computational model of a nitinol stent was used as reference. To translate the computational simulations into physical polymer prototypes, fused deposition modeling (FDM) technology was combined with a commercially available flexible thermoplastic copolyester elastomer (TPC). Copolyesters are an interesting category of bioabsorbable polymers since the ester bonds are susceptible to hydrolysis. The TPC was mechanically characterized by tensile tests and used as input for the computational model. Based on this computationally engineered design, 3D printed stents were obtained and subjected to crush and crimping tests for mechanical validation. Ultimately, an accelerated in vitro degradation test of the polymer stents was performed to assess the biodegradability of the TPC via hydrolysis. Degradation over time was determined via mechanical tensile tests, differential scanning calorimetry (DSC), gel permeation chromatography (GPC), and scanning electron microscopy (SEM).

\section{Materials and Methods}

\section{Computational simulations}

Polymer mechanical characterization. Wires of FlexiFil (Formfutura, Nijmegen, the Netherlands), a flexible TPC filament used for FDM applications, and 3D printed dog bone samples of this material were tested ( $n=3$ each) to take into account the effects of the production procedure. Both groups were evaluated by uniaxial tensile tests in a universal testing machine (Zwicki-Line, Zwick/Roell, Germany) equipped with a $2.5 \mathrm{kN}$ load cell. Samples were loaded at ambient temperature until breaking to assess the mechanical properties. Stress versus strain curves were determined and compared. Finally, the mechanical properties of the $3 \mathrm{D}$ printed samples were used as input for the computational models.

Stent design. The stent geometry used for the computational model is based on a self-expanding laser-cut nitinol stent (pfm medical, Germany) for minimally invasive tissueengineered heart valve implantation in sheep. The stent is composed of a repeating design with 3 rings of 40 struts, connected by tilted bridges. Three-dimensional computational models simulating stent crushing, crimping, and selfexpansion were developed using FE software (ABAQUS 6.14, Dassault Systèmes Simulia Corp. Providence). These models were validated for the nitinol stent in a previous study. ${ }^{9}$
The optimization procedure is an iterative process that consists of adjusting the polymer stent geometry aiming to fulfill two main goals: (i) allow crimpability of the stent to a diameter of $12 \mathrm{~mm}$ consistent with the implantation procedure and (ii) obtain an RF that is comparable to the nitinol stent alternative for the aimed implantation diameter. Based on these considerations, the performance of the polymer prototype can be anticipated by means of computational models in terms of crimp and crush behavior, and compared with the reference nitinol stent. Design parameters such as width, thickness, and number of struts can be adjusted to modify the response of the polymer stent.

Computational crush test. A model of half stent geometry covering $360^{\circ}$ (due to axial symmetry) was used, fixing two nodes in the horizontal direction to avoid rotation. Two plane rigid surfaces were created to simulate parallel plates, initially separated by $31 \mathrm{~mm}$. Surface to surface, finite sliding frictionless contact, enforced by the penalty algorithm, was imposed between the compressing plates (master) and the outer surface stent (slave). Only vertical displacement of the upper plate was allowed, compressing the stent until a clearance of $5 \mathrm{~mm}$ with respect to the lower plate. Afterward, the upper plate returned to its initial position. The vertical reaction force and the displacement of a reference point placed on the upper plate were registered. Due to the symmetry condition, the force was multiplied by two to obtain the final results. Force versus displacement curves of nitinol and polymer stents were compared.

The stent design was modified until the crush response of the polymer stent was equal to or higher than the nitinol stent.

Computational crimping test. To reduce computational time, only a portion of the stent was modeled. The stent geometry was reduced by $1 / n$, where $\mathrm{n}$ is the number of struts. Circumferential symmetry boundary conditions were imposed in agreement with this geometry reduction and axial displacement was prevented by applying a displacement boundary condition at one end of the stent. A cylindrical rigid surface was used to simulate a crimping tool and calculate the RFs. Frictionless contact was assumed between the external surface of the stent and the internal surface of the tool. For transapical implantation of heart valves, $12 \mathrm{~mm}$ delivery complies with clinical relevant administration through the apex of the heart. ${ }^{10}$ Therefore, the stents were crimped by applying radial displacement to the crimping tool until it reached a diameter of $12 \mathrm{~mm}$ to simulate transapical delivery. After crimping, the contact between the stent and the tool was deactivated to allow selfexpansion. The RF of the reduced model was determined by summing the radial reaction forces of all the nodes of the rigid cylinder. To obtain the total RF of the stent, results were multiplied by $\mathrm{n}$ and plotted versus the stent outer diameter (OD).

The polymer stent design was modified to make the stent crimpable, minimizing self-contact of the struts. Force versus diameter curves of nitinol and polymer stents were compared. Furthermore, to illustrate the result of design parameter modifications, the effects of reducing the polymer strut thickness was assessed. 


\section{Prototyping and validation}

Prototyping. Based on the computational model, an optimal stent geometry in terms of RF, crimpability, and recovery was converted to a stereolithography file format via MakerWare software (V 2.4.1.35, MakerBot, New York). The stent design was translated into physical prototypes by using FDM 3D printing technology (Replicator $^{\mathrm{TM}} 2 \mathrm{X}$, MakerBot). Printer settings were specified for a nozzle temperature of $210^{\circ} \mathrm{C}$ and a plateau temperature of $130^{\circ} \mathrm{C}$. Layer height was set at $0.2 \mathrm{~mm}$ with a polymer extruding speed of $90 \mathrm{~mm} / \mathrm{s}$ and a nozzle travel speed of $150 \mathrm{~mm} / \mathrm{s}$. To account for the differences between the computationally derived stent geometry and the 3D printed prototype, a digital image of a stent portion was acquired (30 X, Keyence Digital Microscope VHX-500F) from which a strut profile with more accurate dimensions was sketched and incorporated in the computational models repeating the crush and crimping simulations.

Experimental crush tests. Polymer stent prototypes $(n=5)$ were crushed in a universal testing machine (Zwick/ Roell, Germany) equipped with a $2.5 \mathrm{kN}$ load cell in an ambient environment. The stents were compressed from their nominal diameter until a $5 \mathrm{~mm}$ clearance between plates at a speed of $0.2 \mathrm{~mm} / \mathrm{s}$. Afterward, the top plate returned, at the same speed, to its initial position. Force versus plate displacement was measured. Experimental and computational crush results were compared overlapping the displacement versus force curves.

Experimental crimping tests. Polymer stents $(n=3)$ were crimped in an RF machine (RX 650, Machine Solutions, Inc., Flagstaff, Arizona) at a speed of $0.2 \mathrm{~mm} / \mathrm{s}$. A crimping head composed by 12 movable wedges disposed about a rotational axis, crimped the stents from their nominal diameter until $12 \mathrm{~mm}$. The displacement of the head was later reversed allowing self-expansion of the stent. Experimental and computational crimping results were compared by overlapping the diameter versus force curves.

\section{In vitro degradation characterization}

Differential scanning calorimetry. DSC analyses were used to characterize the melting and crystallization behavior of the polymer, to set the appropriate temperature for en- hanced degradation (823e/700, Mettler-Toledo). A sample was heated to $300^{\circ} \mathrm{C}$ at $20^{\circ} \mathrm{C} / \mathrm{min}$, maintaining this temperature for $5 \mathrm{~min}$, and cooled down to $25^{\circ} \mathrm{C}$ at the same speed. In a subsequent cycle, heating and cooling were performed without holding time. The recorded heat flow was normalized by sample mass. Curves from the first cooling and second heating were computed.

Enhanced hydrolysis. Polymer stents $(n=15)$ were subjected to enhanced hydrolysis in a temperature-controlled water bath, containing demineralized water maintained at $90^{\circ} \mathrm{C}$. Every 7 days, three stents were retrieved, freeze-dried overnight, and stored in an airtight sealed container at room temperature until mechanical testing.

Mechanical analysis. Diamond-shaped samples were cut from the stents and tested uniaxially, by stretching in the circumferential and longitudinal direction $(n=2$ per direction and time point) until break, to assess the loss of mechanical properties by hydrolysis over time.

Gel permeation chromatography. The effect of hydrolysis on the molecular weight of the polymer was assessed via GPC. The GPC measurements $(n=18)$ were performed on a Mixed-D column $(300 \times 7.5$ i.d. $5 \mu \mathrm{m}$ particles, Agilent Technologies) using chloroform at $1 \mathrm{~mL} / \mathrm{min}$ as the mobile phase and a photodiode array (PDA, SPD-M20A, Shimadzu) as the detector. The chromatograms were recorded for the UV absorption of $254 \mathrm{~nm}$. The system was calibrated with polystyrene standards.

Scanning electron microscopy. To investigate the microscopic effects of hydrolysis on the polymer stents, freeze-dried samples $(n=18)$ were analyzed via SEM (UHR-SEM, FEI) at low-vacuum mode with a secondary electron detector at $10 \mathrm{kV}$.

\section{Results}

\section{Polymer stent design}

Mechanical characterization of the polymer. The material properties of the TPC used in this study were determined via uniaxial tensile tests. To assess the influence of $3 \mathrm{D}$ printing on the mechanical properties of the material, stressstrain curves obtained from the polymer wires and 3D printed dog bone samples were compared (Fig. 1A). The printed
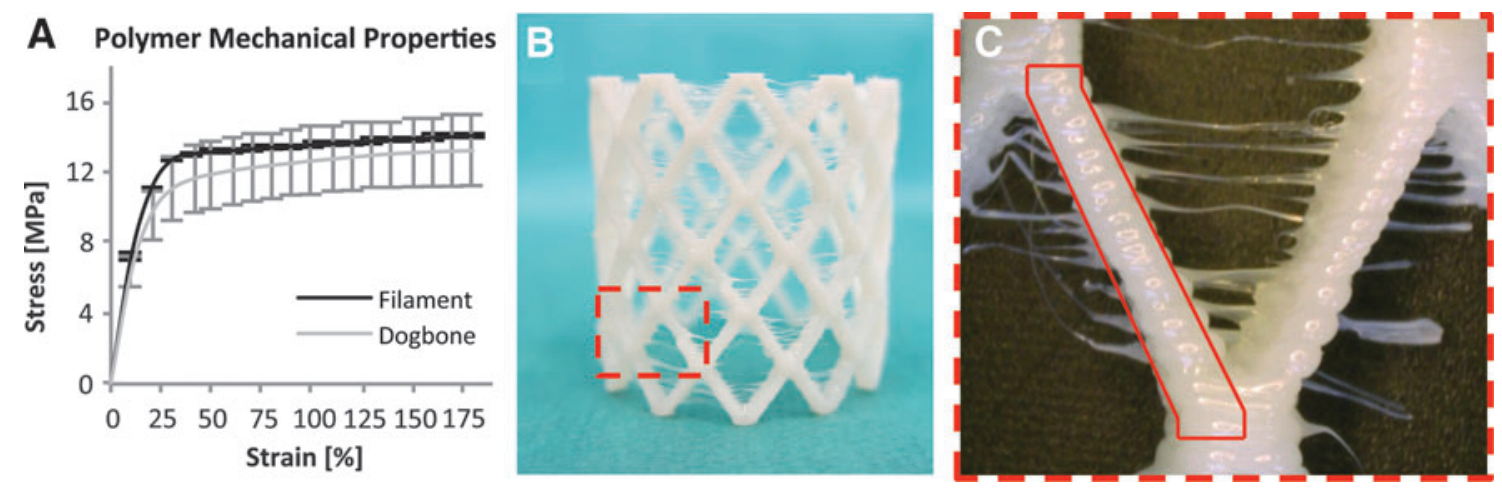

FIG. 1. Polymer stent design. (A) Stress-strain curves of polymer wire and dog bone samples; (B) 3D printed prototype; and $(\mathbf{C})$ computational geometry adjusted considering the prototype dimensions. 
samples deviate from the linear behavior for lower stress values than the wires. However, despite these differences, the decay in tensile properties is not drastic. The averaged stressstrain curve from the printed dog bones was used as input for the simulations.

Polymer stent prototyping. The geometry of a nitinol stent used in preclinical studies ${ }^{4}$ was iteratively modified and implemented in computational crush and crimping tests, with the material properties of the polymer. Adjusting the design parameters to enhance crimpability and RF simultaneously is an extensive process that requires several modifications and ultimately a delicate balance between opposing effects. For example, an increase in the width of the struts increases the $\mathrm{RF}$ of the stent. However, it reduces crimpability and increases the plastic deformation during crimping, leading to smaller diameters after self-expansion. Furthermore, width increase eventually requires a decrease of the number of struts, to fit these dimensional changes along the stent circumference. In addition, this reduction has to be performed in such a way that the total number of struts is dividable by 3 to ensure proper symmetry for valve suturing purposes. Consequently, width increase requires removal of more struts than intended, resulting in a considerable decrease of the RF. An increase in the thickness of the struts generates an increase in the RF without altering the diameter after selfexpansion. Nevertheless, the material and 3D printing technique did not allow a considerable thickness variation in practice. Modifying the length, width, and shape of the bridges that link the struts also contributed to the optimization process.

In general terms, the design that provided the closest performance to a nitinol stent of similar diameter consisted of a stent geometry with 24 struts, 6 rows of struts, an OD of
$30.5 \mathrm{~mm}$, and a length of $30 \mathrm{~mm}$. The prototyped stent, however, slightly differs from the computationally derived geometry due to factors inherent to the $3 \mathrm{D}$ printing manufacturing process (Fig. 1B). To account for these differences, a strut sketch was derived from a digital image of the stent prototype (Fig. 1C) and incorporated in the simulations, which were repeated for a direct comparison with experimental tests.

To illustrate the performance of the optimal design, the 3D printed stent prototype was inserted in a transapical delivery device and deployed in a water bath at $37^{\circ} \mathrm{C}$. The crimping method before implantation consists on placing the stent inside a crimping device (Fig. 2A) and reducing its diameter to $\sim 10 \mathrm{~mm}$ (Fig. $2 \mathrm{~B}$ ) to facilitate insertion in the delivery tool. Once the stent has been transferred from the crimping device to the delivery tool, its OD is $12 \mathrm{~mm}$ (Fig. 2C). To illustrate the transapical stent delivery, the crimped stent was deployed in a water bath at $37^{\circ} \mathrm{C}$ by gradually pushing the stent out of the implantation tool and allowing its selfexpansion (Fig. 2D-H).

\section{Computational analysis and experimental validation}

Crush tests. The simulation shows the presence of plastic deformation at the sides of the stent, creating an oval geometry after load removal (Fig. 3A). Experimental force versus displacement curves, resulting from the crush tests of the polymer 3D printed prototypes, were obtained and compared with the computational crush simulation (Fig. 3B). Experimental results are in good agreement with the computational models.

Crimp tests. The simulation shows a plastic deformation repetitive pattern that is more severe at the zones where two struts meet, causing a reduction in stent diameter after
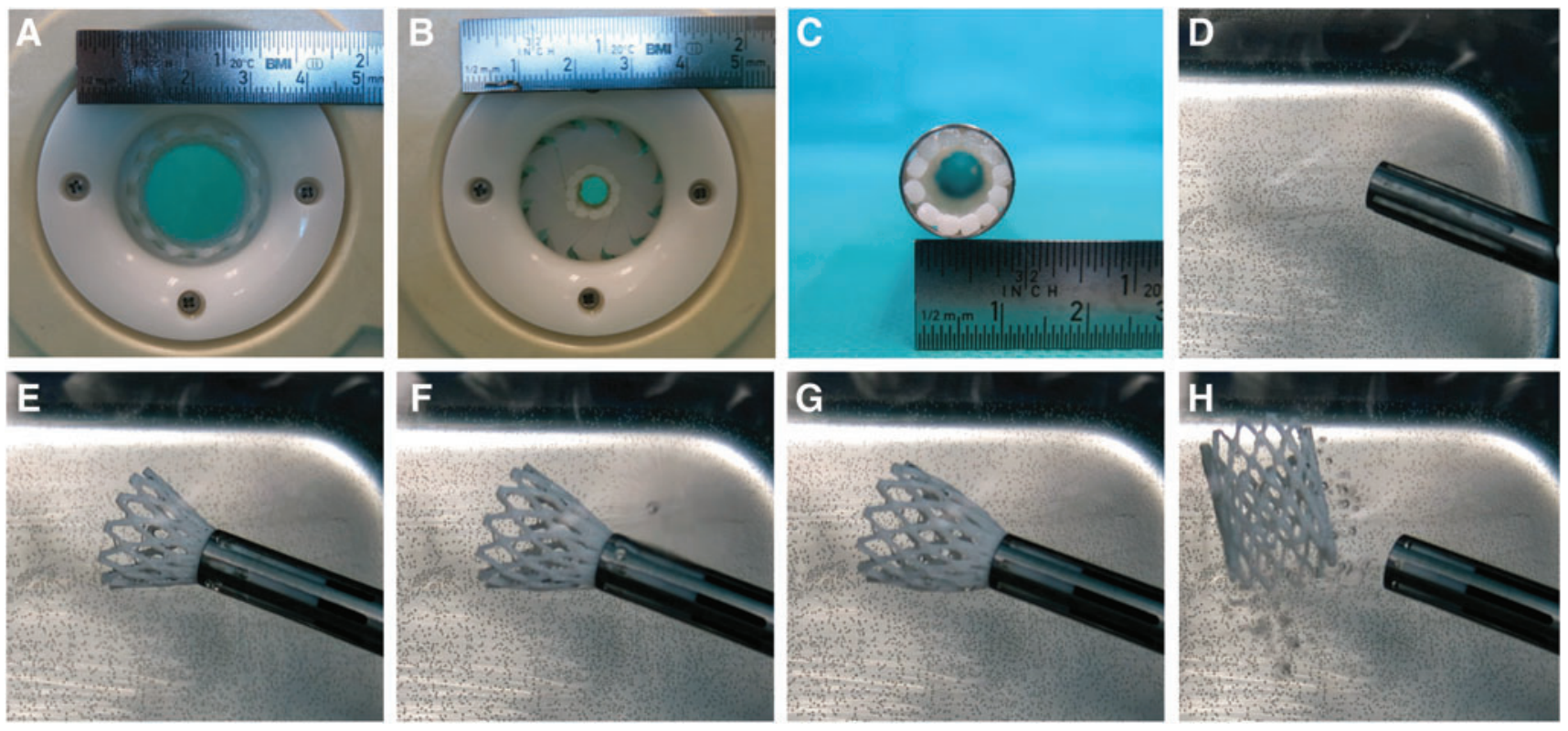

FIG. 2. Crimping and delivery of the 3D printed prototype. (A) 3D printed stent placed inside crimping device. (B) Stent crimping to an approximate diameter of $10 \mathrm{~mm}$. (C) Stent transferred to the transapical delivery device with an internal diameter of $12 \mathrm{~mm}$. (D-H) Self-expansion as the stent is pushed out of the delivery system. 
A

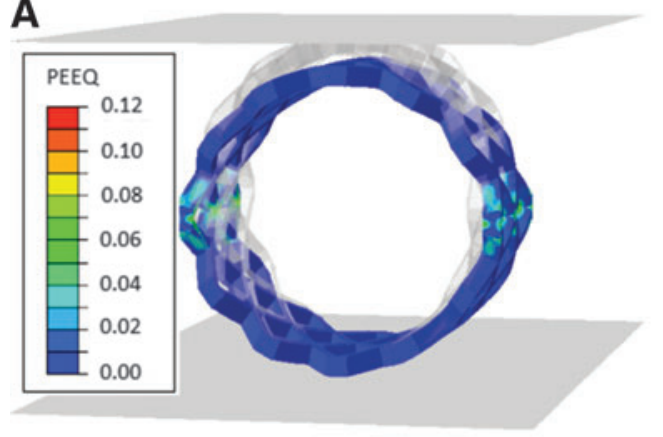

C

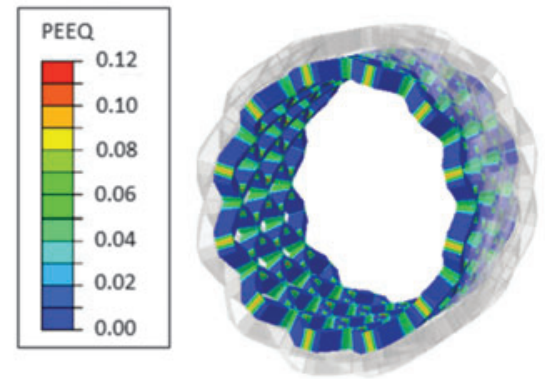

E

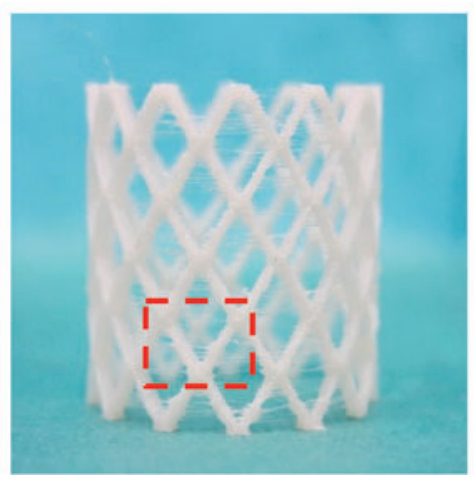

B

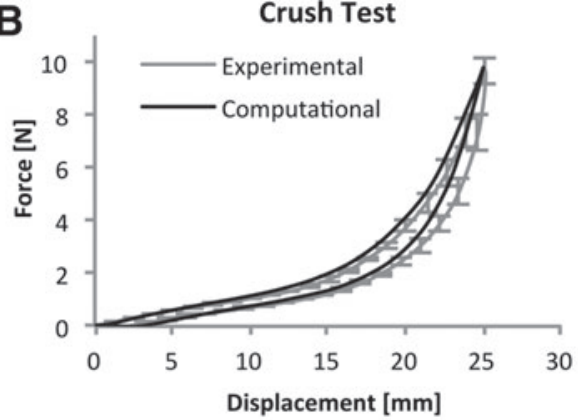

Crimp Test

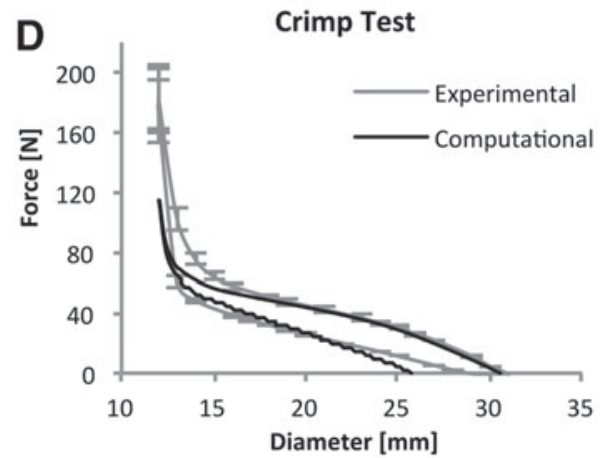

$\mathbf{F}$

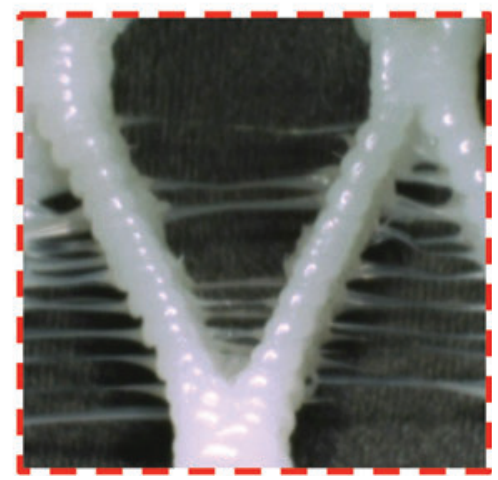

FIG. 3. Crush and crimping tests. (A) Computational crush test (equivalent plastic deformation); (B) computational and experimental force versus displacement curves. Experimental results shown as mean value \pm standard deviation. (C) Computational crimp test (equivalent plastic deformation); (D) computational and experimental force versus diameter curves. Experimental results shown as mean value \pm standard deviation. (E, F) 3 D printed prototype after crimping.

load removal (Fig. 3C). Experimental force versus diameter curves resulting from the crimp tests of the polymer 3D printed prototypes were obtained and compared with the computational crimping (Fig. 3D). Also here, good agreement between computational predictions and experimental results can be observed. However, the diameter after selfexpansion could not be properly captured. Computationally, loading and unloading slopes are coincident. Experimentally, the unloading slope is less steep than the corresponding loading curve. Comparing experimental and computational results, only the loading curves overlap causing a diameter mismatch during unloading. The 3D printed prototype after crimping shows no strut fracture or abnormal strut shape (Fig. 3E, F).

Comparison with nitinol stent. Computational crush polymer stent results were compared with equivalent simulations performed on the nitinol stent that served as a reference. The resulting crush force of the polymer stent is higher than the nitinol stent (Fig. 4A). Due to the presence of plastic deformation in the polymer, the unloading curve does not overlap with the loading curve for low values of plate displacement as it happens with the nitinol stent due to the superelastic nature of this material.

The computational predictions of the crimping behavior of the polymer and nitinol stents were as well compared showing that both alternatives can provide the same RF for a diameter of $22 \mathrm{~mm}$ (Fig. 4B). For smaller diameters, the polymer stent provides even a higher RF than the nitinol stent. The presence of plasticity is not evidenced in the nitinol stent and the initial diameter is recovered upon unloading, whereas the polymer stent experiences a diameter reduction. This situation suggests that if both stents have the same 

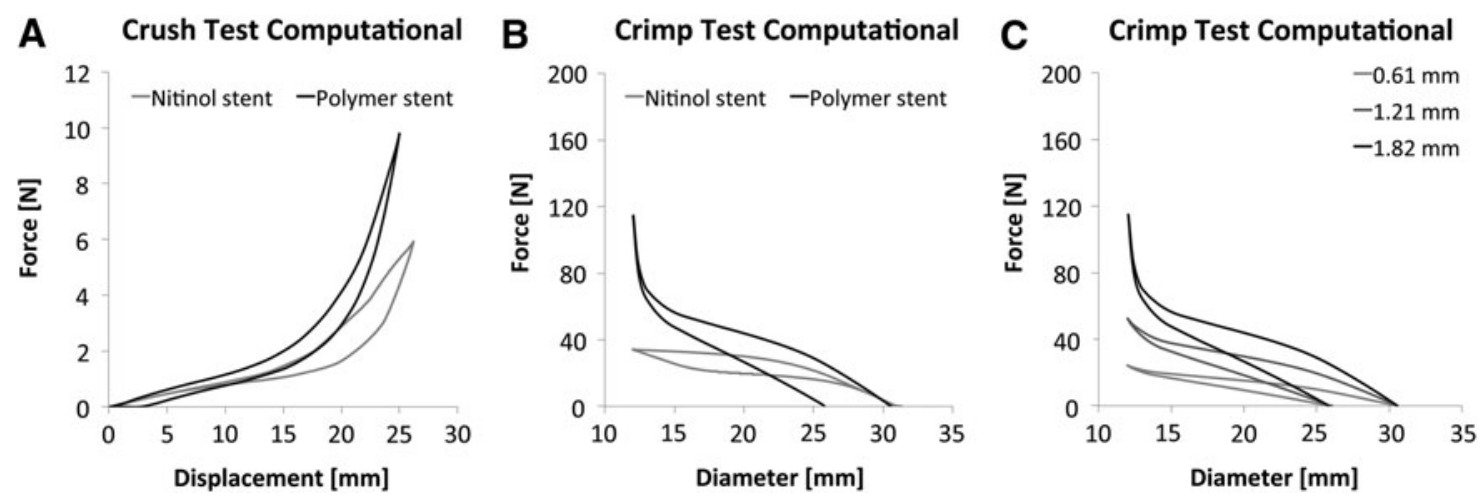

FIG. 4. Computational-based comparison between polymer and nitinol stent. Computational-based comparison between polymer and nitinol stent: (A) crush force versus displacement curve; (B) crimping force versus diameter curve. (C) Crimping force versus diameter curve for different strut thicknesses of the polymer stent.

nominal diameter, the polymer stent would be suitable for implantation in smaller arteries due to its lower diameter after self-expansion. Comparing the unloading curves of both stents, it can be evidenced that the slope of the polymer stent is steeper than the one of the nitinol stent in the vicinity of the intersection point between both lines. To soften the force variation with respect to diameter changes, the thickness of the polymer stent was consequently re- duced. This situation causes a reduction of the RF of the stent while keeping the diameter after self-expansion constant (Fig. 4C).

\section{In vitro degradation characterization}

Differential scanning calorimetry. Polymer phase transition was analyzed by DSC (Fig. 5A). It can be observed that
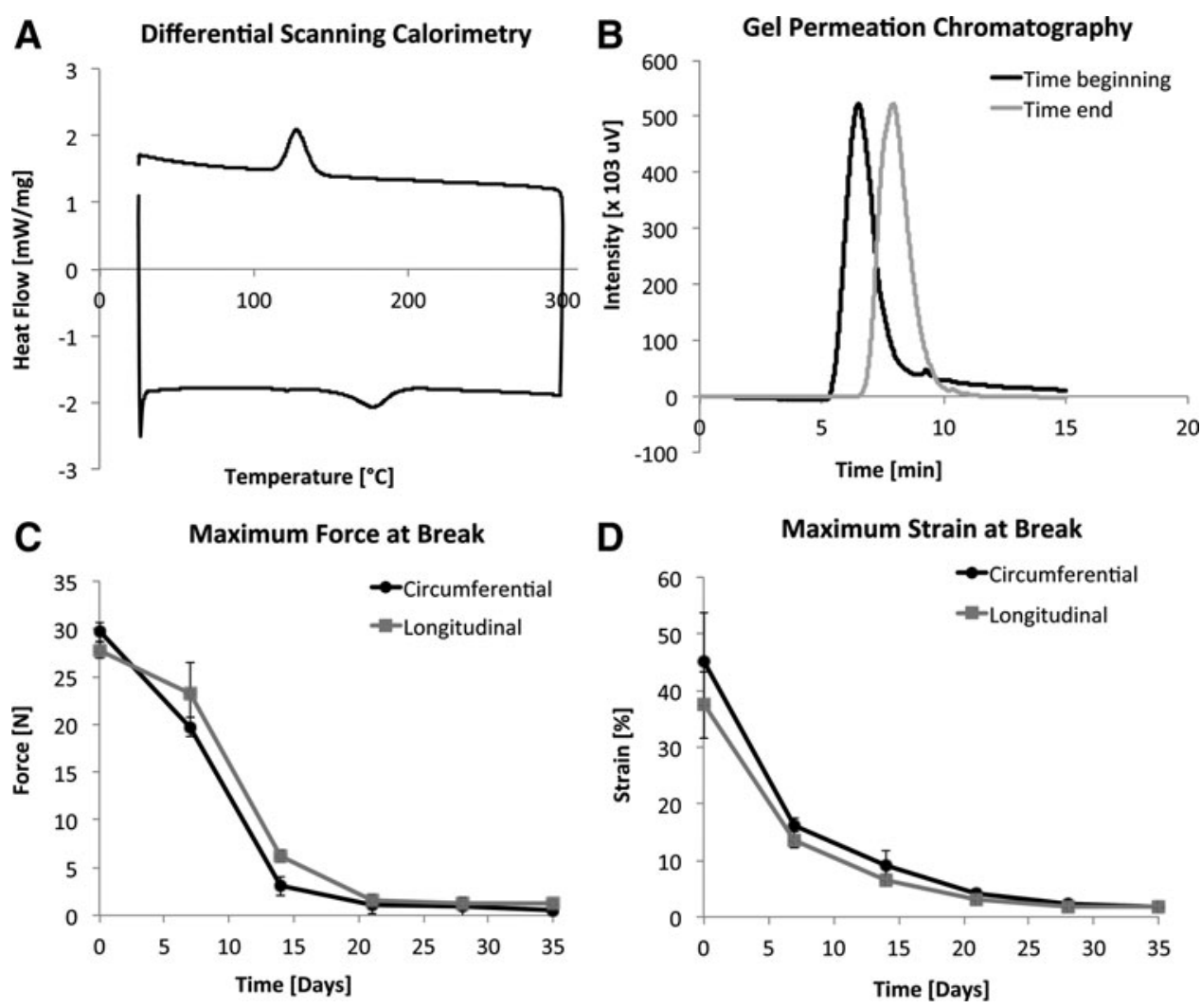

FIG. 5. In vitro characterization of polymer stent. (A) DSC analysis for identification of crystallization and melting phase regions; (B) enhanced degradation at $90^{\circ} \mathrm{C}$ : GPC graphs showing degradation of the polymer in time. The black curve shows the retention at the beginning of the experiment. The gray curve shows the retention at the end of the experiment; (C) maximum force at break in the circumferential and longitudinal direction as a function of time; and (D) maximum strain at break in the circumferential and longitudinal direction as a function of time. Results shown as mean value \pm standard deviation. DSC, differential scanning calorimetry; GPC, gel permeation chromatography. 
this copolyester has a crystallization temperature of $133^{\circ} \mathrm{C}$ and a melting temperature of $184^{\circ} \mathrm{C}$. Therefore, an enhanced in vitro degradation study conducted in $90^{\circ} \mathrm{C}$ water is expected only to enhance hydrolization speed, and not affect phase transition.

Gel permeation chromatography. Figure 5B shows GPC traces of the $3 \mathrm{D}$ printed scaffolds at the beginning of the experiment (t0, black line) and the end of the experiment (tend, pink line). From the graphs it can be observed that the polymer degrades over time, as the retention graph at tend is shifted to longer retention times, indicative for smaller molecular weight.

Mechanical analysis. Diamond-shaped samples obtained from the polymer stent prototype were loaded uniaxially in the circumferential and longitudinal directions showing a decay in the maximal applicable stress over time. After 10 days of enhanced in vitro degradation, mechanical properties dropped to half of the initial force (Fig. 5C). The maximal strains dropped by $50 \%$ around 7 days of enhanced degradation (Fig. 5D). The obtained maximal breaking stresses and strains in the circumferential and longitudinal direction are comparable.

Scanning electron microscopy. The appearance of the surface of the 3D printed polymer stents was smooth before starting the degradation study (Fig. 6A). Over time, the surface roughness increased (Fig. 6B-F). Furthermore, the samples became porous after 14 days of enhanced degradation (Fig. 6C-F).

\section{Discussion}

In this proof-of-concept study, the combination of computational modeling and 3D printing technology was investigated to design large-diameter bioabsorbable polymer stents that can be minimally invasively implanted, allowing growth of the patient. The bottleneck of large-diameter bioabsorbable polymer stent production is the lack of sufficient mechanical properties. ${ }^{11}$ Conventional bioabsorbable polymers, such as poly(lactide acid) PLA, require sophisticated material processing to enable sufficient $\mathrm{RF}^{12}$ and are only applicable to small diameters. Other large-diameter stents deal with stent migration problems due to low RFs. ${ }^{13}$ In this study, we showed that rapid prototyped polymer stents could reach similar levels of RF compared to nitinol alternatives.

FDM printing technology is an evolving and promising approach for polymer processing. It can be used for a broad range of thermoplastic materials where other rapid prototyping methods, such as laser sintering or stereolithography, offer fewer options due to their specific processing technique. ${ }^{14}$ One downside of this method is that the layered nature of the processing technology is less accurate and might present regions with lower mechanical performance compared to the bulk material. However, 3D printing was considered to be an acceptable prototyping technique for the initial phase of bioabsorbable stent design, providing a good compromise between material properties and feasibility to produce prototypes that can be subjected to further tests.

\section{Stent design and host tissue}

Since polymers in general have a lower Young's modulus than metals, geometrical compensation in terms of strut
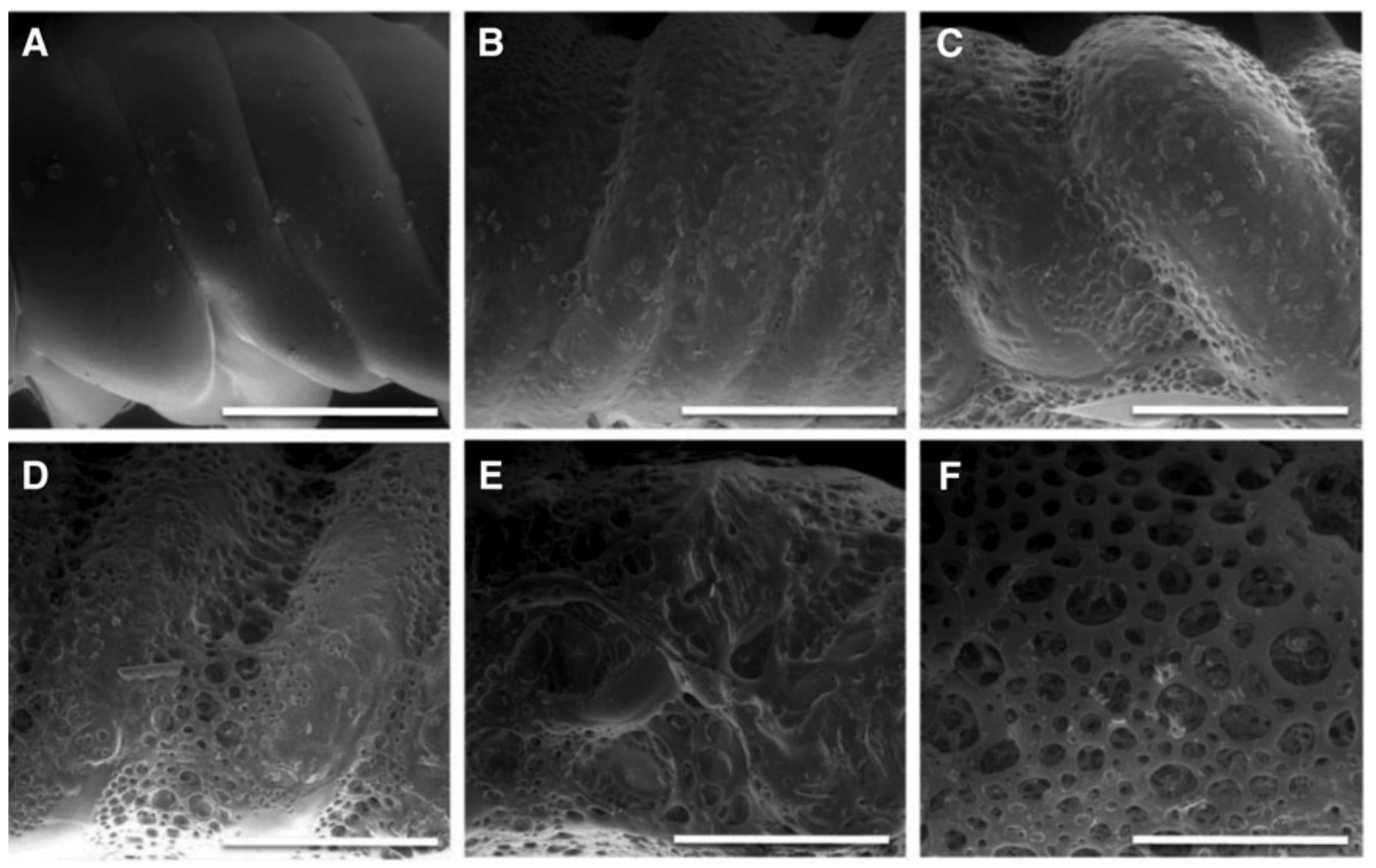

FIG. 6. Surface analysis by SEM during enhanced degradation of polymer stents (bar represents $500 \mu \mathrm{m}$ ). (A) Before degradation (smooth surface); (B, C) increase of surface roughness; (D-F) after 14 days, well-defined pores were distinguished. SEM, scanning electron microscopy. 
thickness and width is required, which could lead to stenosis. ${ }^{15}$ For pediatric applications, where a growing stent is required, further adjustments on the prototype size and thickness need to be performed, to adapt to the size of the patient and the force requirements. The effect of decreasing the strut thickness is depicted in Figure 4C. This technique offers a good alternative to reduce the RF while maintaining the amount of oversizing constant and providing a more gradual force decay during stent deployment. An additional benefit of a lower RF is that after crimping, less force would be required to push the stent out of the delivery device during implantation, by which valvular positioning can be better controlled.

The nitinol stent used as reference for the design of the polymer stent has been successfully used for DTEHV implantation in adult ovine pulmonary arteries of $24-26 \mathrm{~mm}{ }^{4}$ In this diameter range, the corresponding computational RF is $15-17 \mathrm{~N}$ (Fig. 4B). Oversizing of the nitinol stent with respect to the artery is therefore $18.5-28.3 \%$. The polymer stent has shown an experimental diameter loss of $6 \%$ after crimping and self-expansion (Fig. 3B). Aiming to have the same degree of oversizing as the nitinol stent, the polymer stent would be suitable for implantation diameters of 22.4$24.43 \mathrm{~mm}$. For this diameter range, the experimental force of the polymer stents is $13.5-18.9 \mathrm{~N}$, being comparable to the nitinol alternative.

\section{Material properties}

In this proof-of-concept study, a commercially available TPC was used to manufacture 3D printed stents. Apart from proper mechanical characteristics and degradation capacity, special attention has to be paid to the biocompatibility and degradation products of the copolyester. Aromatic polyesters, such as poly(butylene terephthalate) and poly(ethylene terephthalate), which are largely used in the commercial industries $^{16}$ and investigated for their biocompatibility, ${ }^{17,18}$ have excellent mechanical strength, but are more resistant to hydrolytic degradation. ${ }^{19}$ Biocompatible aliphatic polyesters such as PLA and poly(caprolactone) ${ }^{20}$ have good biodegradative properties in terms of hydrolysis, but lack proper mechanical strength. ${ }^{21}$ Blending aromatic and aliphatic building blocks seems feasible and might provide the ability to tune mechanical properties and degradation. ${ }^{22}$ The TPC choice for this proof-of-concept study was based on its intrinsic mechanical properties and its potential to undergo hydrolytic degradation. Nevertheless, this material is not biocompatible and therefore cannot be considered for translational studies.

\section{Degradation}

The speed at which hydrolysis of polyesters occurs in vivo depends on multiple factors, such as monomer composition, relative humidity, temperature, and bioactivity of present enzymes. ${ }^{23}$ Besides hydrolytic degradation, oxidative and enzymatic degradation also contributes to in vivo polymer degradation. ${ }^{24}$ However, to what extent these pathways are affecting the total degradation speed has to be further investigated. The TPC selected for this proof-of-concept study has shown to be susceptible to hydrolysis, which in combination with 3D FDM printing can be an interesting method to facilitate adaptation of the stent to growth of the patient. A

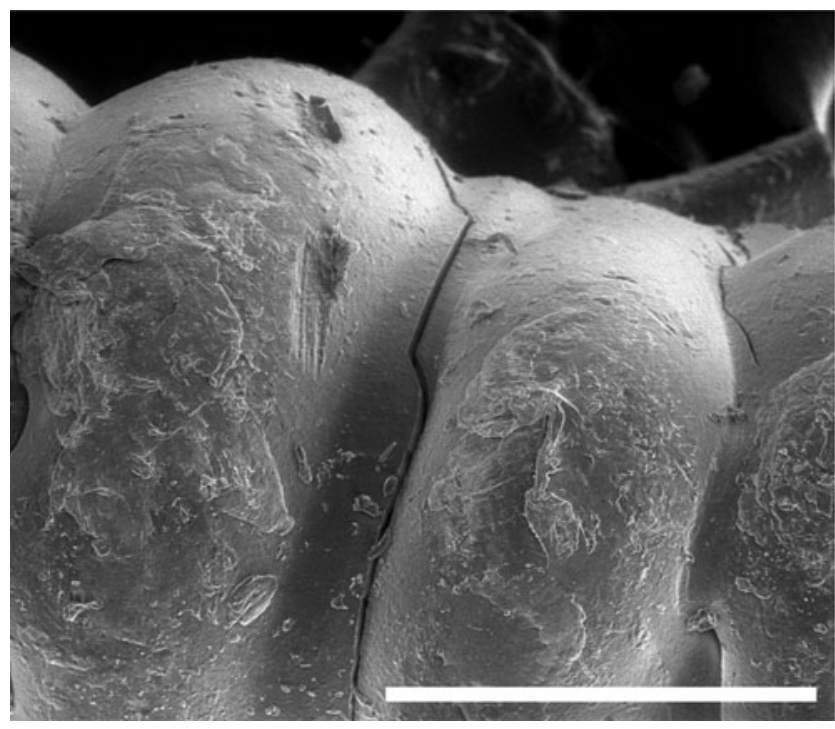

FIG. 7. Surface analysis by SEM after 3 weeks of enhanced degradation (bar represents $500 \mu \mathrm{m}$ ). Crack located at the layered interphase.

consequence inherent to the use of FDM, which involves heating of the material to create small flattened strings enabling the deposition of molten layers, is the creation of a clearly visible layered structure (Fig. 1C). This layered profile can initiate predefined breaking points over time. Within this study, we evidenced the presence of cracks located at the layered interface as depicted in one of our SEM images after 3 weeks of accelerated degradation (Fig. 7). This finding suggests that even if the stent does not fully degrade, a concept prototype that accommodates for growth of the patient by enabling hydrolysis-induced breaking points could be envisioned. These breaking points, combined with the increasing fragility of the material, could disrupt the stent pattern under physiological cyclic loading, which could lead to an opened structure that does not hamper growth of the artery.

Based on our previous in vivo experience, ${ }^{4}$ the stent and the heart valve are fully integrated within the native wall after 8-16 weeks of implantation. When the stent is incorporated by the host tissue, stent fragments that may arise from the degradation process are prevented from entering the blood stream. After tissue encapsulation, cell-mediated degradation of polymer remnants proceeds to contribute to the removal of polymeric residues. Macrophages could phagocyte the remnants or form giant body cells to engulf the remnants. On the contrary, granulocytes such as mast cells, eosinophils, and basophils could secrete cytokines, chemokines, and matrix metalloproteinases to enhance polymer degradation. ${ }^{25,26}$

\section{Quality of the $3 D$ printing method}

The low resolution of the manufacturing technique imposes certain limitations that can ultimately affect the performance of the stent. For the scope of this study, the performance of the stent is defined as its ability to successfully deliver a DTEHV in a minimally invasive manner for preclinical evaluation. This means that the stent has to be able 
to comply with transapical requirements (i.e., crimping diameter of $12 \mathrm{~mm}$, self-expansion diameter in agreement with the size of the host tissue) and provide sufficient RF to remain anchored (which was considered the force of the nitinol stent used as reference). The combination of material and 3D printing technique selected for this study limits the capacity to perform accurate thickness changes. In addition, reducing the number of struts below 21 causes disrupted strut patterns when the prototype is printed. These impediments limit the capacity to adjust the crimpability and RF of the prototype.

Once the most optimal design has been reached, the 3D printed prototype differs from the theoretical design. The combination of the selected material and 3D printing method results in a relatively low resolution of the prototyped struts, which deviates from the straight theoretical/computational strut shape (Fig. 1C, red contour) defining a more curved and nonuniform profile in practice. In addition, after deposition of one layer, the molten material can be carried along with the $3 \mathrm{D}$ printing nozzle to the next strut, creating thin wires that connect the struts (Fig. 1C, thin wires on black background). The imperfections induced by the manufacturing method have a negligible effect on the crush force of the stent (Fig. 3B) and a more considerable effect on the RF of the stent during the crimping phase. For crimping diameters smaller than $20 \mathrm{~mm}$ (Fig. 3D), RF measurement curves show that it requires a higher crimping force to reduce the diameter of the stent in practice. The reason for this deviation is attributed to the thin wires that connect the struts (Fig. 1C), which represent a physical obstacle that offers some resistance to crimping. Nevertheless, it is worth mentioning that the crimping force does not define the performance of the stent, which is characterized by the RF during self-expansion. For diameters comparable to the implantation site, the theoretical and experimental self-expansion RF does not show differences. Despite the fact that these discrepancies do not introduce significant changes in the performance of the stent, they introduce poor-quality features that should not be present in further versions of the prototype.

\section{Future directions}

In the future, further effort will be dedicated to implement or develop clinically suitable polymers that are biocompati- ble and simultaneously comply with $3 \mathrm{D}$ printing requirements. An interesting material platform for 3D printing applications can be built based on the use of supramolecular polymers. By means of supramolecular chemistry, aromatic and aliphatic components could be equipped with quadruple hydrogen bonding moieties, creating building blocks that enable independent tuning of mechanical properties and degradation speed. An additional benefit of supramolecular moieties is that only a small increase in temperature is needed to overcome the bonds between building blocks, and material characteristics are fully restored after cooling. ${ }^{27}$ These features can ease rapid prototyping processing. For future studies, biocompatible stents, 3D printed with copolymers with similar mechanical properties as the TPC used in this study but different degradation speeds, should be evaluated in vivo in an animal model. Particular focus should be made on biocompatible, bioabsorbable materials that degrade after 16 weeks of exposure to the host environment ${ }^{4}$ to make sure that the prosthesis has been fully integrated within the host tissue before the degradation process begins.

A future study with a newly synthesized material should be conducted at $37^{\circ} \mathrm{C}$ to determine the effect of hydrolysis on polymer degradation. However, in vivo polymer degradation does not solely rely on hydrolysis. In addition, oxidative degradation caused by cellular components and cyclic mechanical load would influence the degradation speed of the stent. It is for this reason that for a better evaluation, additional oxidative processes should be incorporated in an experimental setup. ${ }^{28}$ However, despite how complete in vitro degradation studies might be, in vivo situations could still lead to different results. ${ }^{29}$

One possible way to reduce the presence of imperfections in the strut profile could be to modify the composition of the material reducing, for example, its viscosity. Nevertheless, this can drastically modify the overall mechanical behavior of the material and the stent design would have to be subsequently adjusted. Mechanical grinding alternatives were not pursued in this study since the imperfections are located in different planes (tangent to the inner and outer surface, and throughout the thickness at the strut edges) and due to the potential introduction of external particles arising from abrasive methods. Chemical etching seems to be one practical way to eliminate these imperfections without having a deep influence on the performance of the designed stent. In
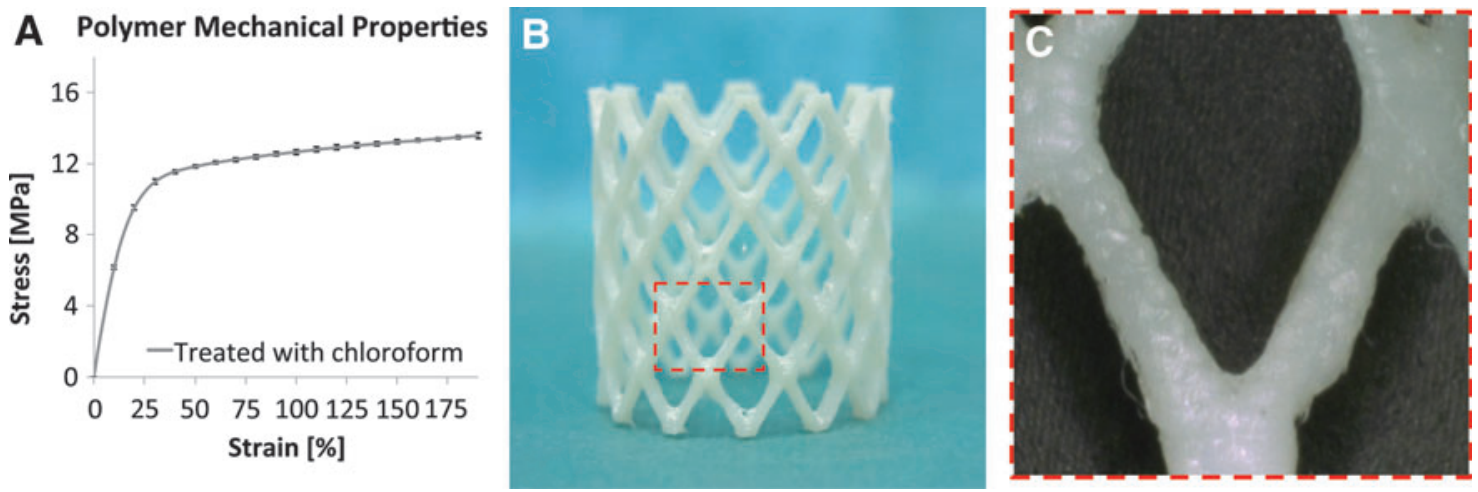

FIG. 8. Chemical etching: (A) Stress-strain curves of 3D printed dog bone samples treated with chloroform; (B) 3D printed stent prototype after chloroform treatment; and (C) flattening of the strut profile and reduction of the thin wires that connected the struts. 
the context of this proof-of-concept study, three dog bone samples and one 3D printed stent were immersed in chloroform for $60 \mathrm{~s}$ to reduce the presence of imperfections. To assess the influence of chemical etching on the mechanical properties of the material, stress-strain curves obtained from 3D printed dog bone samples treated with chloroform were obtained (Fig. 8A). A comparison with Figure 1A shows that the mechanical properties of the $3 \mathrm{D}$ printed dog bone samples were not affected by the exposure to chloroform. Furthermore, the standard deviation has been reduced considerably after the chemical treatment. With respect to the shape of the struts, immersion in chloroform flattened the curved and nonuniform strut profile and dissolved the thin wires that connected the struts (Fig. 8B, C). Therefore, chemical post-treatment of 3D printed stents could be considered an interesting possibility to improve the quality of the struts.

\section{Conclusion}

In order for DTEHVs to be minimally invasively implanted and have the capacity to grow with the patient, stents with growth potential have to be developed. In this proof-ofprinciple study, it was shown that computational-based 3D printed self-expandable and biodegradable polymer stents, with a reasonable degree of plastic deformation and RFs comparable to nitinol stents, can be successfully designed. The computational simulations have demonstrated the capacity to build models with realistic outcomes, based on uniaxial material characterization. In addition, the FDM 3D printing technique is a promising manufacturing technique to translate computational models into physical prototypes that can be used to assess the mechanical performance of stent designs. Furthermore, the copolyester materials used in this study have shown to possess biodegradation potential via hydrolysis.

\section{Acknowledgments}

The authors thank Martin van Drongelen (Department of Mechanical Engineering, Section Polymer Technology, Eindhoven University of Technology) for performing the differential scanning calorimetry analysis. This work was financially supported by the European Union's Seventh Framework Programme (FP7/2007-2013) under grant agreement number 242008 (LifeValve).

\section{Author Disclosure Statement}

No competing financial interests exist.

\section{References}

1. Sun JC, Davidson MJ, Lamy A, et al. Antithrombotic management of patients with prosthetic heart valves: Current evidence and future trends. Lancet 2009;374:565-576.

2. Yacoub MH, Takkenberg JJM. Will heart valve tissue engineering change the world? Nat Clin Pract Cardiovasc Med 2005;2:60-61.

3. Hammermeister K, Sethi GK, Henderson WG, et al. Outcomes 15 years after valve replacement with a mechanical versus a bioprosthetic valve: Final report of the Veterans Affairs randomized trial. JACC 2000;36:1152-1158.

4. Driessen-Mol A, Emmert MY, Dijkman, PE, et al. Transcatheter implantation of homologous "off-the-shelf" tissue- engineered heart valves with self-repair capacity. J Am Coll Cardiol 2014;63:1320-1329.

5. Kornowski R, Hong MK, Tio FO, et al. In-stent restenosis: Contributions of inflammatory responses and arterial injury to neointimal hyperplasia. JACC 1996;31:224-230.

6. Mayoral W, Fleischer D, Salcedo J, et al. Nonmalignant obstruction is a common problem with metal stents in the treatment of esophageal cancer. Gastroint Endosc 2000;51: $556-559$.

7. Wang Z, Li N, Li R, et al. Biodegradable intestinal stents: A review. Prog Natl Sci Mater Int 2014;24:423-432.

8. Ormiston JA, Serruys PWS. Bioabsorbable coronary stents. Circ Cardiovasc Interv 2009;2:255-260.

9. Cabrera MS, Oomens CWJ, Biaaijens FPT. Understanding the requirements of self-expandable stents for heart valve replacement: radial force, loop force, and equilibrium, J Biomech 2017;68:252-264.

10. Schmidt D, Dijkman PE, Driessen-Mol A, et al. Minimallyinvasive implantation of living tissue engineered heart valve. A comprehensive approach from autologous vascular cells to stem cells. J Am 2010;56:510-520.

11. Waksman R. Update on bioabsorbable stents: From bench to clinical. J Intervent Cardiol 2006;19:414-421.

12. Serruys PW, Ormiston JA, Onuma Y. A bioabsorbable everolimus-eluting coronary stent system (ABSORB): 2year outcomes and results from multipleimaging methods. Lancet 2009;373:897-910.

13. Černá M, Köcher M, Válek V, et al. Covered biodegradable stent: New therapeutic option for the management of esophageal perforation or anastomotic leak. Cardiovasc Intervent Radiol 2011;34:1267-1271.

14. Capel AJ, Edmondson S, Christie SDR, et al. Design and additive manufacture for flow chemistry. Lab Chip 2013; 13:4583-4590.

15. Hara H, Nakamura M, Palmaz JC, et al. Role of stent design and coatings on restenosis and thrombosis. Adv Drug Deliv Rev 2006;58:377-386.

16. Müller RJ, Kleeberg I, Deckwer WD, et al. Biodegradation of polyesters containing aromatic constituents. J. Biotechnol 2001;86:87-95.

17. Gupta B, Plummer C, Bisson I, et al. Plasma-induced graft polymerization of acrylic acid onto poly(ethylene terephthalate) films: Characterization and human smooth muscle cell growth on grafted films. Biomaterials 2009;23: 863-871.

18. Prowans P, Fray El M, Slonecki J. Biocompatibility studies of new multiblock poly(ester-ester)s composed of poly(butylene terephthalate) and dimerized fatty acid. Biomaterials 2002;23:2973-2978.

19. Vroman I, Tighzert L. Biodegradable polymers. Materials 2009;2:307-344.

20. Generali M, Dijkman PE, Hoerstrup SP. Bioresorbable scaffolds for cardiovascular tissue engineering. EMJ Int Cardiol 2014;1:91-99.

21. Vert M. Aliphatic polyesters: Great degradable polymers that cannot do everything. Biomacromolecules 2004;6: 538-546.

22. Chen Y, Tan L, Chen L, Yang Y, Wang X. Study on biodegradable aromatic/aliphatic copolyesters. Braz J Chem Eng 2008;25:321-335.

23. Gunatillake PA, Adhikari R. Biodegradable synthetic polymers for tissue engineering. Eur Cell Mater 2003;5:1-16.

24. Azevedo HS, Reis RL. Understanding the Enzymatic Degradation of Biodegradable Polymers and Strategies to 
Control Their Degradation Rate. Biodegradable Systems in Tissue. Boca Raton: CRC Press, 2005.

25. Anderson JM, McNally AK. Biocompatibility of implants: Lymphocyte/macrophage interactions. Semin Immunopathol 2011;33:221-233.

26. van Loon S, Smits A, Driessen-Mol A, et al. The immune response in in situ tissue engineering of aortic heart valves. In: Aikawa E. (Ed.); Calcific Aortic Valve Disease. West Palm Beach: Intech 2013; pp. 207-245.

27. Bosman AW, Sijbesma RP, Meijer EW. Supramolecular polymers at work. Mater Today 2004;7:34-39.

28. Brugmans MCP, Söntjens SHM, Cox MAJ, et al. Hydrolytic and oxidative degradation of electrospun supramolecular biomaterials: In vitro degradation pathways. Acta Biomater 2015;27:21-31.
29. Mishra A, Seethamraju K, Delaney J, et al. Long-term in vitro hydrolytic stability of thermoplastic polyurethanes. J Biomed Mater Res A 2015;103:3798-3806.

Address correspondence to: María Sol Cabrera

Department of Biomedical Engineering Group of Soft Tissue Biomechanics and Tissue Engineering Eindhoven University of Technology Eindhoven $5600 \mathrm{MB}$ The Netherlands

E-mail: m.s.cabrera@tue.nl 